\title{
A PREVALÊNCIA DE ANSIEDADE E FATORES ASSOCIADOS EM MÉDICOS DE UMA UNIVERSIDADE PÚBLICA BRASILEIRA NA PANDEMIA COVID-19
}

\section{THE PREVALENCE OF ANXIETY AND ASSOCIATED FACTORS IN PHYSICIANS AT A BRAZILIAN PUBLIC UNIVERSITY IN THE COVID-19 PANDEMIC}

\author{
Gabriel Chiquetto Kava'*, Tatiana Menezes Garcia Cordeiro', Lara Simone Messias Floriano', \\ Nilmara Duran', Guilherme Arcaro', Rafaela Olívia Moreno Marinho'. \\ 1 Universidade Estadual de Ponta Grossa, Departamento de Medicina, Ponta Grossa, Paraná, Brasil \\ "Autor Correspondente: Endereço: Rua Valério Ronchi, 160, Bloco 14 Apartamento 201 - CEP 84030-320; \\ Telefone: (42) 99951-7235; e-mail: gabrielchiquettokava@gmail.com
}

\section{RESUMO}

Com a atual pandemia de COVID-19, os profissionais de saúde diretamente ligados ao atendimento de pacientes suspeitos e infectados vêm apresentando prejuízos não só em saúde física, mas também mental. O constante medo psicológico e as grandes demandas físicas e emocionais são fatores estressores que os trabalhadores médicos têm de enfrentar, o que pode contribuir para o surgimento ou agravamento de transtornos psiquiátricos, particularmente os transtornos de ansiedade. O objetivo do presente estudo foi identificar a prevalência e fatores associados à ansiedade em profissionais médicos que atuam na docência, médicos que atuam exclusivamente na assistência e médicos residentes de um Hospital Universitário Regional do Sul do Brasil, que é referência no atendimento a casos de COVID-19 em sua região. Trata-se de um estudo observacional transversal, no qual foi utilizada a plataforma Google Forms para coleta de dados, e o conteúdo respondido foi um questionário sociodemográfico, de condições de hábitos de vida e a escala de Transtorno Geral de Ansiedade (GAD-7). Ao todo, 51 profissionais de 97 constatados completaram a pesquisa. A maior parte da amostra foi constituída por homens $(64,7 \%)$, a média de idade foi de 40,7 anos, $47,1 \%$ trabalham há mais de 10 anos e $31,4 \%$ relataram alguma alteração do sono. A prevalência de ansiedade foi de $25,49 \%$, com predomínio feminino $(53,85 \%)$, mediana de idade de 34 anos, solteira $(53,85 \%)$, branca $(69,23 \%)$. No presente estudo, pôde-se constatar que menor tempo de carreira, laborações associadas à COVID-19, residir sozinho e a não prática de atividade física regular foram fatores associados à ansiedade.

Palavras-chave: Pandemias; Infecções por Coronavírus; Saúde mental; Ansiedade.

\section{ABSTRACT}

With the current COVID-19 pandemic health professionals working directly in the care of suspected and infected patients have suffered some unbalance of both their physical and mental health. The constant psychological fear and the great physical and emotional demands are stressors that medical workers have to face, which can contribute to the emergence or worsening of psychiatric disorders, particularly anxiety disorders. The objective of this study was to identify the prevalence and factors associated with anxiety in medical professionals who work in teaching, doctors who work exclusively in assistance and residents of a Regional University Hospital in Southern Brazil, which is a reference in the treatment of cases of COVID-19 in the region. This is a cross-sectional 
observational study that used the Google Forms platform to collect data, a sociodemographic questionnaire was applied, whose content included living conditions and the General Anxiety Disorder (GAD-7) scale. Fifty-one professionals out of the 97 contacted completed the survey. Most of the respondents were men (64.7\%), the average age was 40.7 years, $47.1 \%$ worked for more than 10 years and $31.4 \%$ reported some sleep disorder. The prevalence of anxiety was $25.49 \%$, with a predominance of single (53.85\%), white (69.23\%) women (53.85\%), the median age was 34 years. The results of the study showed shorter time in the career, activities associated with COVID-19, living alone and the lack of regular physical activity were factors associated with anxiety.

Keywords: Pandemics; Coronavirus infections; Mental Health; Anxiety.

\section{INTRODUÇÃO}

A pandemia da COVID-19, nome da síndrome respiratória ocasionada pelo novo coronavírus (SARS-CoV-2), foi inicialmente detectada em 2019 na cidade de Wuhan, província de Hubei na China ${ }^{1,2}$. A transmissão foi expansiva e em apenas um mês a doença foi considerada uma emergência de saúde pública pela Organização Mundial da Saúde (OMS) e foi declarada pandemia em 11 de março de $2020^{3}$.

Diante dessa situação, os profissionais de saúde que atuam diretamente com a crise passaram a lidar com o número progressivo de casos confirmados e suspeitos, com a carga de trabalho excessiva, com a exaustão física e emocional, com a falta de equipamentos de proteção individual e de insumos, com o elevado risco de autoinfecção e de infectar amigos e parentes. Tais fatores podem contribuir para a sobrecarga na saúde mental. ${ }^{1,4,5}$ Desde a eclosão da pandemia COVID-19, vários estudos investigaram o estado mental das equipes médicas e confirmaram a presença de altos índices de ansiedade, estresse, insônia e depressão nessa população ${ }^{1,6,7}$. O constante medo psicológico da pandemia e as grandes demandas físicas e emocionais são fatores estressores que os trabalhadores médicos têm de enfrentar, podendo contribuir para o surgimento ou agravamento de transtornos psiquiátricos, particularmente os transtornos de ansiedade, sendo que o Brasil lidera o mundo em termos de prevalência ${ }^{4,8}$.

Os transtornos de ansiedade referem-se a um grupo de transtornos mentais caracterizados por sentimentos de ansiedade e medo, incluindo transtorno de ansiedade generalizada (TAG), transtorno do pânico, fobias, transtorno de ansiedade social, transtorno obsessivo-compulsivo (TOC) e transtorno de estresse pós-traumático (TEPT) ${ }^{9}$. A intensidade elevada da ansiedade, além de trazer para o médico problemas psíquicos, pode trazer prejuízo ao trabalho e ao relacionamento pessoal. Seu desempenho profissional também pode ser comprometido em virtude de problemas do sono, fadiga ou de baixa concentração, ameaçando a qualidade do atendimento e a segurança dos pacientes ${ }^{10}$.

Perante as evidências que apontam claramente a vulnerabilidade mental da classe, o CRM-PR adotou atendimentos psiquiátricos gratuitos via telefone por voluntários cadastrados na associação paranaense de psiquiatria, o atendimento não substitui a consulta presencial e tem como objetivo auxiliar os profissionais da linha de frente a lidar com as angústias ${ }^{11}$.

A cidade polo da $3^{\text {a }}$ Regional de Saúde do Paraná é Ponta Grossa e o Hospital Universitário Regional é a referência no atendimento a casos de Covid-19, contando com um Comitê de Enfrentamento da doença, responsável por pontuar as principais diretrizes, estabelecer protocolos e fazer o gerenciamento 
de leitos. Uma equipe multiprofissional, a partir da triagem, define se o paciente irá para a enfermaria ou UTI e estabelece a melhor conduta de tratamento. A organização do hospital foi elaborada para obter insumos, EPIs e para promover capacitação para que, dessa forma, o suporte ao paciente seja o melhor possível e possa-se manter a segurança, saúde e integridade dos profissionais.

Assim, com o possível impacto negativo da pandemia na saúde mental dos médicos, o presente estudo teve por objetivo identificar a prevalência e fatores associados à ansiedade em médicos que atuam na docência, médicos que atuam exclusivamente na assistência e médicos residentes de um Hospital Universitário Regional do Sul do Brasil, no contexto atual da pandemia COVID-19.

\section{MATERIAIS E MÉTODOS}

Trata-se de um estudo transversal exploratório, de análises quantitativas e descritivas, guiado pela ferramenta STROBE, realizado com médicos que atuam na docência, médicos que atuam exclusivamente na assistência e pelos médicos residentes da instituição de referência ao enfrentamento da COVID-19, na cidade de Ponta Grossa - PR. A coleta de dados foi realizada inteiramente por meio da plataforma Google Forms e ocorreu no interstício compreendido entre agosto e setembro de 2020, disponibilizada ao público de interesse nas redes sociais das quais fazem parte.

A amostra foi selecionada por conveniência a fim de obter um recenseamento da situação dos profissionais. Foram considerados como critérios de inclusão: ser médico e ter registro ativo no CRM-PR. Foram excluídos trabalhadores que estavam em licença médica, em férias ou que estavam afastados. A coleta de dados foi desenvolvida junto aos médicos por livre aceitação em participação do estudo, mediante aceite da pesquisa por meio de um Termo de Consentimento Livre e Esclarecido anexo ao questionário.

Desenvolveu-se um questionário estruturado, contendo características sociodemográficas, condições de hábitos de vida e um questionário de avaliação de ansiedade. O instrumento utilizado para identificação de ansiedade foi a versão brasileira validada da escala de Transtorno Geral de Ansiedade (GAD-7) traduzida para a língua portuguesa pela Pfizer. A GAD-7 é composta por sete itens, dispostos em uma escala de quatro pontos: 0 (nenhuma vez) a 3 (quase todos os dias), com pontuação que varia de 0 a 21, ao medir frequência de sinais e sintomas de ansiedade nas últimas duas semanas. Considera-se indicador positivo de sinais e sintomas de transtornos de ansiedade valor igual ou maior que $10^{12,13,14}$.

Considerou-se como variável dependente a ocorrência de ansiedade e como variáveis independentes foram consideradas as características sociodemográficas, de atuação e categorização profissional, e condições de hábitos de vida por intermédio dos seguintes elementos: idade, sexo, estado civil, raça/etnia, renda, nível de formação, presença de outros vínculos empregatícios, setor em que trabalha, tempo de atuação, executa laborações associadas à COVID-19, quantas pessoas residem com o participante, prática de atividades físicas regulares, presença de comorbidades, tabagismo, etilismo, consumo durante a pandemia, alteração do sono e medicamentos de uso contínuo. Buscou-se identificar distintos fatores para verificar se algum deles se associa positivamente com a ansiedade.

Para testar a associação entre a variável dependente "presença de ansiedade" e as variáveis independentes, realizou-se a análise bivariada pelo teste qui-quadrado. As variáveis que apresentaram valor de $\mathrm{P} \leq 0,05$ na análise foram classificadas como diferença significativa. Os dados foram expressos como frequências e percentagens; como mediana (M) \pm desvio padrão (DP). Os dados foram analisados utilizando o software estatístico Statistical Package for the Social Sciences 20 (SPSS $\left.{ }^{\circledR}\right)$. 
O projeto vinculado ao Programa UEPG Abraça, registrado com CAAE 21415319.4.0000.0105, obteve aprovação do Comitê de Ética em Pesquisa em Seres Humanos da UEPG sob número de parecer 3.983.323 e da Diretoria Acadêmica e o Centro de Estudos, Pesquisa e Desenvolvimento Humano do HURCG.

\section{RESULTADOS}

Entre os 97 profissionais, 54 médicos docentes e 43 médicos residentes, 51 aceitaram participar da pesquisa, constituindo uma taxa de participação de 52,5\%. As perdas decorreram da recusa por parte dos médicos em participar da pesquisa, da possível não visualização do formulário nos veículos on-line e da própria rotina médica durante a pandemia COVID-19 ( $\mathrm{n}=46)$.

O presente estudo identificou uma prevalência de 25,49\% de ansiedade nos profissionais médicos em relação ao instrumento utilizado. A amostra foi composta por 35,3\% de mestres, 25,5\% de residentes, $21,6 \%$ de doutores, $9,8 \%$ de especialistas e 7,8\% de graduados. A média de idade foi de 40,7 anos, variando entre 24 e 72 anos, composta por 64,7\% de homens, 92,2\% de cor branca, 64,7\% de casados e 52,9\% com renda superior a 15 salários mínimos.

Em relação ao tempo de atuação dos profissionais, 47,1\% trabalham há mais de 10 anos, 9,8 \% trabalham entre 5 e 10 anos, 33,3\% de 1 a 5 anos e 9,8 \% há menos de 1 ano. No que se refere ao trabalho, 51\% não possuem outro vínculo empregatício e 56,9\% exercem laborações relacionadas à COVID-19, abrangendo tenda de triagem, terapia intensiva e enfermaria, atendimento em call center, atendimento em pronto-socorro e supervisão, atenção primária (UBS), gestão e assistência médica.

Quanto à moradia, 47,1\% residem com 1 ou 2 pessoas, sendo que 62,7\% com cônjuge. Além disso, $68,6 \%$ dos médicos praticam atividades físicas regulares e 51\% não possuem comorbidades, $17,6 \%$ apresentam dislipidemia, 11,8\% hipertensão arterial sistêmica, 11,8\% transtorno bipolar e 3,9\% asma (gráfico 3). O uso de medicamento contínuo está presente em $57,7 \%$ dos profissionais. Com relação ao tabagismo e etilismo, 94,2\% dos médicos não fumam e 75\% ingerem bebida de teor alcoólico; entre os tabagistas, $42,9 \%$ relataram aumento do uso do tabaco durante a pandemia. No que diz respeito ao sono, 32,7\% afirmaram apresentar alguma alteração durante a pandemia, sendo que desses 64,7\% relataram insônia. As características sociodemográficas e condições de hábitos de vida associadas à ansiedade, avaliada conforme o resultado obtido pela aplicação da Escala de Transtorno Geral de Ansiedade (GAD-7) obtido com a aplicação do questionário, estão descritas na Tabela 1.

Tabela 1 - Perfil sociodemográfico e condições de hábitos de vida de profissionais médicos com ansiedade que atuam na assistência ou ensino, em Ponta Grossa, Paraná, Brasil, 2020

\begin{tabular}{|c|c|c|c|c|c|}
\hline \multicolumn{2}{|l|}{ Variáveis } & $\operatorname{Sim} n(\%)$ & Não n(\%) & Total n(\%) & p valor \\
\hline \multicolumn{2}{|c|}{ Presença de Ansiedade } & $13(25,49)$ & $38(74,51)$ & $51(100,00)$ & \\
\hline \multirow{2}{*}{ Sexo } & Masculino & $6(46,15)$ & $26(68,42)$ & $32(62,75)$ & 0,343 \\
\hline & Feminino & $7(53,85)$ & $12(31,58)$ & $19(37,25)$ & \\
\hline Idade & $M, S D$ & $34( \pm 10,11)$ & $44( \pm 12,55)$ & $42( \pm 12,39)$ & 0,086 \\
\hline \multirow{2}{*}{ Estado civil } & Solteiro & $7(53,85)$ & $11(28,95)$ & $18(35,29)$ & \\
\hline & Casado & $6(46,15)$ & $27(71,05)$ & $33(64,71)$ & 0,105 \\
\hline \multirow{2}{*}{ Cor } & Branca & $9(69,23)$ & $38(100,00)$ & $47(92,16)$ & \\
\hline & Parda & $4(30,77)$ & $0(0,00)$ & $4(7,84)$ & $<0,001$ \\
\hline
\end{tabular}


continuação

\begin{tabular}{|c|c|c|c|c|c|}
\hline \multicolumn{2}{|l|}{ Variáveis } & $\operatorname{sim} n(\%)$ & Não n(\%) & Total n(\%) & p valor \\
\hline \multirow{4}{*}{ Renda } & Até 5 salários mínimos & $1(7,69)$ & $2(5,26)$ & $3(5,88)$ & \\
\hline & De 5-10 salários mínimos & $2(15,38)$ & $4(10,52)$ & $6(11,76)$ & 0,080 \\
\hline & De 10-15 salários mínimos & $7(53,85)$ & $8(21,05)$ & $15(29,41)$ & \\
\hline & $\begin{array}{l}\text { Mais de } 15 \text { salários } \\
\text { mínimos }\end{array}$ & $3(23,08)$ & $24(63,17)$ & $27(52,94)$ & \\
\hline \multirow{5}{*}{ Nível de Formação } & Graduado & $4(30,77)$ & $0(0,00)$ & $4(7,84)$ & \\
\hline & Especialista & $1(7,69)$ & $4(10,52)$ & $5(9,80)$ & 0,007 \\
\hline & Residente & $4(30,77)$ & $9(23,68)$ & $13(25,49)$ & \\
\hline & Mestre & $3(23,08)$ & $15(39,47)$ & $18(35,29)$ & \\
\hline & Doutor & $1(7,69)$ & $10(26,31)$ & $11(21,57)$ & \\
\hline \multirow{2}{*}{$\begin{array}{l}\text { Possui outro vínculo } \\
\text { empregatício }\end{array}$} & Sim & $9(69,23)$ & $16(42,10)$ & $25(49,02)$ & 0,091 \\
\hline & Não & $4(30,77)$ & $22(57,90)$ & $26(50,98)$ & \\
\hline \multirow{4}{*}{ Tempo de Atuação } & Menos de 1 ano & $2(15,38)$ & $3(7,89)$ & $5(9,80)$ & \\
\hline & De 1 a 5 anos & $6(46,15)$ & $11(28,95)$ & $17(33,33)$ & 0,334 \\
\hline & De 5 a 10 anos & $0(0,00)$ & $5(13,16)$ & $5(9,80)$ & \\
\hline & Acima de 10 anos & $5(38,47)$ & $19(50,00)$ & $24(47,06)$ & \\
\hline \multirow{2}{*}{$\begin{array}{l}\text { Executa laborações } \\
\text { associadas à COVID-19? }\end{array}$} & Sim & $12(92,31)$ & $17(44,74)$ & $29(56,96)$ & \\
\hline & Não & $1(7,69)$ & $21(55,26)$ & $22(43,14)$ & 0,003 \\
\hline \multirow{6}{*}{$\begin{array}{l}\text { Quantas pessoas residem } \\
\text { com o participante? }\end{array}$} & 1 & $2(15,38)$ & $11(28,95)$ & $13(25,49)$ & \\
\hline & 2 & $1(7,69)$ & $10(26,31)$ & $11(21,57)$ & \\
\hline & 3 & $4(30,77)$ & $5(13,16)$ & $9(17,65)$ & \\
\hline & 4 & $1(7,69)$ & $7(18,42)$ & $8(15,68)$ & 0,049 \\
\hline & 5 ou mais & $0(0,00)$ & $2(5,26)$ & $2(3,92)$ & \\
\hline & Sozinho & $5(38,47)$ & $3(7,89)$ & $8(15,68)$ & \\
\hline \multirow{2}{*}{ Mora com Namorado? } & Sim & $1(7,69)$ & $2(5,26)$ & $3(5,88)$ & 0,748 \\
\hline & Não & $12(92,31)$ & $36(94,74)$ & $48(94,12)$ & \\
\hline \multirow{2}{*}{ Mora com Pai ou Mãe? } & Sim & $1(7,69)$ & $6(15,79)$ & $7(13,73)$ & 0,464 \\
\hline & Não & $12(92,31)$ & $32(84,21)$ & $44(86,27)$ & \\
\hline \multirow{2}{*}{ Mora com irmão? } & Sim & $0(0,00)$ & $3(7,89)$ & $3(5,88)$ & 0,296 \\
\hline & Não & $13(100,00)$ & $35(92,11)$ & $48(94,12)$ & \\
\hline \multirow{2}{*}{ Mora com cônjuje? } & Sim & $6(46,15)$ & $27(71,05)$ & $33(64,71)$ & \\
\hline & Não & $7(53,85)$ & $11(28,95)$ & $18(35,29)$ & 0,105 \\
\hline \multirow{2}{*}{ Mora sozinho? } & Sim & $5(38,47)$ & $3(7,89)$ & $8(15,68)$ & 0,009 \\
\hline & Não & $8(61,53)$ & $35(92,11)$ & $43(84,32)$ & \\
\hline \multirow{2}{*}{ Mora com sogro (a)? } & Sim & $0(0,00)$ & $3(7,89)$ & $3(5,88)$ & 0,296 \\
\hline & Não & $13(100,00)$ & $35(92,11)$ & $48(94,12)$ & \\
\hline \multirow{2}{*}{ Mora com filhos? } & Sim & $5(38,47)$ & $16(42,10)$ & $21(41,18)$ & 0,818 \\
\hline & Não & $8(61,53)$ & $22(57,90)$ & $30(58,82)$ & \\
\hline \multirow{2}{*}{ Mora com amigo? } & Sim & $0(0,00)$ & $2(5,26)$ & $2(3,92)$ & 0,399 \\
\hline & Não & $13(100,00)$ & $36(94,74)$ & $49(96,08)$ & \\
\hline \multirow{2}{*}{$\begin{array}{l}\text { Pratica atividade física } \\
\text { regular? }\end{array}$} & Sim & $5(38,47)$ & $30(78,95)$ & $35(68,63)$ & 0,007 \\
\hline & Não & $8(61,53)$ & $8(21,05)$ & $16(31,37)$ & \\
\hline
\end{tabular}

continua 
conclusão

Variáveis

$\operatorname{Sim} n(\%)$

Não n(\%)

Total $n(\%)$

p valor

\begin{tabular}{|c|c|c|c|c|c|}
\hline \multirow{6}{*}{$\begin{array}{l}\text { Apresenta alguma } \\
\text { comorbidade? }\end{array}$} & $\begin{array}{l}\text { Não apresenta } \\
\text { comorbidade }\end{array}$ & $4(30,77)$ & $22(57,89)$ & $26(50,98)$ & 0,172 \\
\hline & Diabetes Mellitus & $0(0,00)$ & $1(2,63)$ & $1(1,96)$ & \\
\hline & $\begin{array}{l}\text { Hipertensão arterial } \\
\text { sistêmica }\end{array}$ & $1(7,69)$ & $4(10,53)$ & $5(9,80)$ & \\
\hline & Dislipidemia & $2(15,38)$ & $6(15,79)$ & $8(15,68)$ & \\
\hline & Outras & $2(15,38)$ & $3(7,89)$ & $5(9,80)$ & \\
\hline & Transtorno psiquiátrico & $4(30,77)$ & $2(5,26)$ & $6(11,76)$ & \\
\hline \multirow{2}{*}{ É tabagista? } & Sim & $1(7,69)$ & $2(5,26)$ & $3(5,88)$ & 0,748 \\
\hline & Não & $12(92,31)$ & $36(94,74)$ & $48(94,12)$ & \\
\hline \multirow{2}{*}{$\begin{array}{l}\text { Ingere bebidas de teor } \\
\text { alcoólico? }\end{array}$} & $\operatorname{sim}$ & $11(84,62)$ & $27(71,05)$ & $38(74,51)$ & 0,333 \\
\hline & Não & $2(15,38)$ & $11(28,95)$ & $13(25,49)$ & \\
\hline \multirow{2}{*}{$\begin{array}{l}\text { Apresenta / apresentou } \\
\text { alguma alteração de sono } \\
\text { durante a pandemia? }\end{array}$} & Sim & $4(30,77)$ & $12(31,58)$ & $16(31,37)$ & \\
\hline & Não & $9(69,23)$ & $26(68,42)$ & $35(68,63)$ & 0,957 \\
\hline \multirow{2}{*}{$\begin{array}{l}\text { Utiliza medicamentos de } \\
\text { uso contínuo? }\end{array}$} & Sim & $10(76,92)$ & $19(50,00)$ & $29(56,96)$ & \\
\hline & Não & $3(23,08)$ & $19(50,00)$ & $22(43,14)$ & 0,091 \\
\hline
\end{tabular}

$M$ (mediana), SD (desvio padrão)

Fonte: $O$ autor.

\section{DISCUSSÃO}

A presente pesquisa identificou uma prevalência de 25,49\% de ansiedade nos profissionais médicos. Uma meta-análise sobre o impacto da pandemia COVID-19 na saúde mental dos trabalhadores de saúde de países asiáticos indicou uma prevalência semelhante de mais de $20 \%$ de ansiedade, com diferenças de gênero e ocupação. A prevalência calculada combinada de ansiedade foi de 23,21\%, com 13 estudos transversais que relataram a prevalência de depressão, ansiedade ou insônia durante a pandemia ${ }^{15}$.

Estudos que utilizaram o mesmo instrumento de avaliação desta pesquisa apresentaram uma maior prevalência combinada. Quatro estudos com amostras entre 1257 a 5062 trabalhadores de saúde (19,8\% até 39,2\% da amostra composta por médicos) utilizaram a escala GAD-7 com uma prevalência combinada de $36,92 \%^{1,16-18,15}$. Potenciais diferenças se devem a uma aplicação de pontuação de corte mais alta neste estudo e a uma amostra menor, além de diferentes fatores sociais, culturais e econômicos. Em uma revisão integrativa, as taxas variaram em 20,1\% a 44,6\% de ansiedade, o que mostra o considerável efeito da crise em toda a população de profissionais de saúde e que as taxas são amplamente comparáveis ${ }^{5}$. A prevalência de ansiedade observada nesta pesquisa também esteve em consonância com o estudo de Zhu Z et al. (2020), no qual foi encontrado por meio da mesma escala uma taxa de ansiedade entre os trabalhadores de saúde de $24,06 \%{ }^{16}$.

Em relação ao perfil sociodemográfico dos profissionais com ansiedade $(25,49 \%)$, pode -se observar que houve uma predominância feminina (53,85\%) com a mediana de idade de 34 anos com um desvio padrão de $(10,11)$, solteira $(53,85 \%)$, branca $(69,23 \%)$. O predomínio das mulheres também foi observado em diferentes estudos e se acredita estar relacionado à propensão preestabelecida do 
sexo ao distúrbio, além de características mais sensíveis ao sofrimento ${ }^{15,19}$. Da mesma forma, Du J et al. (2020) encontraram sintomas de ansiedade mais comuns em mulheres ${ }^{20}$.

Quanto ao tempo de atuação profissional, mais da metade (61,53\%) dos médicos que indicaram ansiedade possuía um tempo de atuação pequeno, menos de 1 ano (15,38\%) e de 1 a 5 anos $(46,15 \%)$, sendo cerca de $1 / 3$ dos profissionais residentes e $30,77 \%$ graduados $(P=0,007)$, tal fato pode estar relacionado com o período hostil de início de carreira (internato e residência), que expõe o profissional a níveis elevados de estresse e uma alta demanda de trabalho tanto física quanto emocional. Estudos estimam que mais de três quartos dos médicos residentes apresentam esgotamento profissional ${ }^{21}$. Além disso, percebe-se também que os médicos em início de carreira estão mais preocupados e pensativos com suas perspectivas futuras. O medo de falhar e frustrações quanto ao reconhecimento profissional são sentimentos comuns que, se não forem bem administrados, podem trazer consequências ruins à saúde do médico. Em contrapartida, no presente estudo um nível de formação maior apresentou menores níveis de ansiedade $(\mathrm{P}=0,007)$ e isso se deve possivelmente a um rearranjo mais equilibrado da carreira profissional ${ }^{22}$.

A atividade médica diretamente relacionada à COVID-19 está associada a maiores índices de ansiedade $(\mathrm{P}=0,003)$, sendo que nesta pesquisa $92,31 \%$ dos médicos que apresentaram ansiedade executam laborações associadas diretamente à COVID-19. Vários estudos já foram realizados avaliando a saúde dos trabalhadores de saúde durante as epidemias da Síndrome Respiratória Aguda Grave - SARS, Síndrome Respiratória do Oriente Médio -MERS e do Novo coronavírus - COVID-19 e evidenciaram que os profissionais de saúde foram relacionados a taxas significativas de ansiedade,

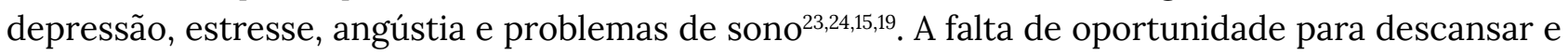
dormir adequadamente associada à falta de EPIs e à alta carga de trabalho e exaustão emocional pode exacerbar os impactos na saúde mental ${ }^{24,25}$.

No que se refere ao número de pessoas que residem com os médicos, o indivíduo que reside sozinho apresentou-se neste estudo como um fator associado à ansiedade $(\mathrm{P}=0,009)$. Em um estudo transversal realizado no estágio inicial da pandemia na Espanha, por meio de uma pesquisa online com 3480 pessoas, a solidão foi relacionada a um maior impacto psicológico, sendo um dos preditores principais para ansiedade, depressão e estresse pós-traumático..$^{18}$ Aqueles que se comunicam menos fora do ambiente de trabalho possuem menor apoio nos períodos de descanso em suas residências e isso pode gerar um desequilíbrio emocional ${ }^{22}$.

Com relação ao sono, 31,4\% dos profissionais afirmaram apresentar alguma alteração durante a pandemia, sendo mais frequente a insônia. Em um estudo de Lai J et al. (2020), 34\% dos médicos e enfermeiras de 34 hospitais da China apresentaram insônia ${ }^{1}$. Em outra pesquisa que avaliou a qualidade do sono na pandemia, um em cada quatro profissionais de saúde tinha problemas de sono ${ }^{17}$. É evidente que a qualidade do sono interfere de forma efetiva na saúde dos indivíduos e está provavelmente associada ao tempo não suficiente para descansar, às aflições e aos temores pela presença da pandemia.

No que diz respeito à prática de atividade físicas, os profissionais de saúde que apresentavam hábitos regulares foram associados a um menor risco para ansiedade $(\mathrm{P}=0,007)$. A atividade física pode ser fator-chave para ajudar a população a tolerar melhor os períodos de pandemia, tanto no nível mental quanto físico ${ }^{25}$. No estudo de Zhu Z et al. (2020), também foi encontrada essa associação, em que a execução de exercícios pode suavizar o impacto psicológico causado por traumas, bem como corrobora para uma rotina estável e equilibrada ${ }^{16}$. 
Apesar do presente estudo não apresentar associação entre comorbidades e a ansiedade, o maior risco poderia se associar ao receio da contaminação e dos desfechos negativos do grupo de risco para a COVID-19. Cerca de 60\% dos médicos que apresentaram ansiedade possuíam alguma comorbidade, sendo que por volta de $1 / 3$ relataram ter algum transtorno psiquiátrico. Nesse sentido, nosso estudo foi incapaz de distinguir sintomas preexistentes de novos sintomas relacionados à saúde mental.

Enfim, o impacto negativo da pandemia na saúde mental e física, sobretudo nos níveis de ansiedade, afeta a atenção dos trabalhadores médicos, a compreensão e a tomada de decisão, o que pode atrapalhar a luta contra o coronavírus. Além disso, é capaz de ter um efeito duradouro em suas vidas, podendo associar a progressão da ansiedade e depressão com o Burnout, uma reação emocional crônica caracterizada por desmotivação, desinteresse e um mal-estar geral na relação com o trabalho $26,27,28$.

As limitações do estudo incluem ausência de acompanhamento longitudinal dos profissionais de saúde, incapacidade em distinguir sintomas preexistentes de novos sintomas relacionados à saúde mental e o viés de seleção relacionado ao desejo de participar. Portanto, as implicações psicológicas de longo prazo nessa população merecem uma investigação mais aprofundada.

\section{CONCLUSÃO}

Com os dados levantados neste estudo, pode-se concluir que foram identificados altos níveis de ansiedade em médicos durante a pandemia. Executar laborações associadas à COVID-19, residir sozinho, menor tempo de carreira e ausência de atividade física regular foram fatores associados à ansiedade.

Por isso, estes profissionais devem receber atenção especial nos cuidados de saúde mental, pois se encontram em situação de vulnerabilidade, na qual esses sintomas e transtornos podem ser aumentados ou estabelecidos, sendo importante para sua própria saúde, para a qualidade do atendimento ao paciente e para o combate à pandemia. Este estudo pode servir como evidência importante para direcionar a promoção do bem-estar mental e identificar estratégias de enfrentamento para prevenir e combater os possíveis impactos da pandemia COVID-19 na saúde mental dos profissionais médicos.

\section{REFERÊNCIAS}

1. Lai J, Ma, S, Wang Y, Cai Z, Hu J, Wei N, et al. 2020. Factors associated with mental health outcomes among health care workers exposed to coronavirus disease 2019. JAMA Netw Open. 2020; 23;3(3):e203976-e203976. doi: 10.1001/jamanetworkopen.2020.3976

2. Zhu N, Zhang D, Wang W, Li X, Yang B, Song J, et al. A novel coronavirus from patients with pneumonia in China, 2019. New Engl. J. Med. 2020;382:727-733. doi: 10.1056/NEJMoa2001017

3. World Health Organization (WHO). Statement on the Selcond Meeting of the International Health Regulations (2005) Emergency Committee Regarding the Outbreak of Novel Coronavirus (2019-nCoV). [Internet]. 2020. [cited 2020 Sep 29]. Available from: https://www.who.int/news/ item/30-01-2020-statement-on-the-second-meeting-of-the-international-health-regulations(2005)-emergency-committee-regarding-the-outbreak-of-novel-coronavirus-(2019-ncov) 
4. Ornell F, Halpern SC, Kessler FHP, Narvaez JC M. The impact of the COVID-19 pandemic on the mental health of healthcare professionals. Cad. Saúde Pública. 2020; 36(4):e00063520. https:// doi.org/10.1590/0102-311x00063520

5. Prado AD, Peixoto BC, da Silva AMB, Scalia LAM. A saúde mental dos profissionais de saúde frente à pandemia do COVID-19: uma revisão integrativa. Revista Eletrônica Acervo Saúde. 2020;(46):e4128. https://doi.org/10.25248/reas.e4128.2020

6. Huang JZ, Han, MF, Luo TD, Ren, AK, Zhou XP. Mental health survey of 230 medical staff in a tertiary infectious disease hospital for COVID-19. Chin. J. Industrial Hygiene Occupational Diseases. 2020;38(0):E001. doi: 10.3760/cma.j.cn121094-20200219-00063

7. Liu Z, Han B, Jiang R, Huang Y, Ma C, Wen J, et al. Mental health status of doctors and nurses during COVID-19 epidemic in China. SSRN Electron. J. 2020; https://doi.org/10.2139/ssrn.3551329

8. De Souza IM, Machado-De-Souza JP. Brazil: world leader in anxiety and depression rates. Rev. Bras. Psiquiatr. 2017; 4(39):384. https://doi.org/10.1590/1516-4446-2017-2300

9. World Health Organization (WHO). Depression and other common mental disorders: Global health estimates. [Internet]. 2017 [cited 2020 Oct 11]. Available from: apps.who.int/iris/ bitstream/handle/10665/254610/1/WHO-MSD-MER-2017.2-eng.pdf.

10. Quek TT, Tam WW, Tran BX, Zhang M, Zhang Z, Ho CSH, et al. The Global Prevalence of Anxiety Among Medical Students: A Meta-Analysis. Int J Environ Res Public Health. 2019;16(15):2735. doi:10.3390/ijerph16152735

11. Conselho Regional de Medicina do Estado do Paraná (CRM-PR). Profissionais que atuam no combate à COVID-19 podem solicitar atendimento psiquiátrico gratuito. [Internet]. 2020. [cited 2020 Sep 29]. Available from: https://www.crmpr.org.br/Profissionais-queatuam-no-combate-a-COVID19-podem-solicitar-atendimento-psiquiatrico-gra-1 1-53865. shtml\#: :text=Preocupada\%20com\%20a\%20sa\%C3\%BAde\%20mental,meio\%20de\%20 volunt\%C3\%A 1 rios\%20j\%C3\%A 1\%20cadastrados.

12. Spitzer RL, Kroenke K, Williams JBW, Löwe B. A brief measure for assessing generalized anxiety disorder: the GAD-7. Archives of Internal Medicine. 2006;10(166):1092-1097. doi: 10.1001/ archinte.166.10.1092

13. Kroenke K, Spitzer RL, Williams JBW, Monahan PO, Löwe B. Anxiety disorders in primary care: prevalence, impairment, comorbidity, and detection. Annals of Internal Medicine. 2007; (146):317-325. doi: 10.7326/0003-4819-146-5-200703060-00004

14. Mapi Research Institute. Certificate of linguistic validation certificate: general anxiety disorder-7 (GAD-7). 2006; Lyon, FR: Mapi Research Institute.

15. Pappa S, Ntella V, Giannakas T, Giannakoulis VG, Papoutsi E, Katsaounou P. Prevalence of depression, anxiety, and insomnia among healthcare workers during the COVID-19 pandemic: A systematic review and meta-analysis. Brain Behav Immun. 2020;88:901-907. doi: 10.1016/j. bbi.2020.05.026

16. Zhu Z, XU S, Wang H. COVID-19 in Wuhan: Immediate Psychological Impact on 5062 Health Workers. EClinicalMedicine. 2020. doi: 10.1016/j.eclinm.2020.100443.

17. Huang Y, Zhao N. Generalized anxiety disorder, depressive symptoms and sleep quality during COVID-19 outbreak in China: a web-based cross-sectional survey. Psychiatry Research. 2020; 288:1 12954. M. doi:10.1101/2020.02.19.20025395. 
18. Zhang C, Yang L, Liu S, Ma S, Wang Y, Cai Z, et al. Survey of insomnia and related social psychological factors among medical staff involved in the 2019 novel coronavirus disease outbreak. Front. Psychiatry. 2020;1 1:306. doi:10.3389/fpsyt.2020.00306

19. González-Sanguino C, Ausín B, Castellanos MA. Mental health consequences during the initial stage of the 2020 Coronavirus pandemic (COVID-19) in Spain. Brain, Behavior, and Immunity. 2020; 87:172-176. doi:10.1016/j.bbi.2020.05.040

20. Du J, Dong L, Wang T, Yuan C, Fu R, Zhang L, et al. Psychological symptoms among frontline healthcare workers during COVID-19 outbreak in Wuhan. General Hospital Psychiatry. 2020. https://doi.org/10.1016/j.genhosppsych.2020.03.011

21. Gracino ME, Zitta ALL, Mangilini OC, Massuda EM. A saúde física e mental do profissional médico: uma revisão sistemática. Saúde debate. 2016;1 10(40):244-263. http://dx.doi. org/10.1590/0103-1104201611019

22. Song X, Fu W, Liu X, Luo Z, Wang R, Zhou N, et al. Mental health status of medical staff in emergency departments during the Coronavirus disease 2019 epidemic in China. Brain, Behavior, and Immunity. 2020; 88: 60-65. doi: 10.1016/j.bbi.2020.06.002

23. Salazar de Pablo G, Vaquerizo-Serrano J, Catalan A, Arango C, Moreno C, Ferre F, et al. Impact of coronavirus syndromes on physical and mental health of health care workers: systematic review and meta-analysis. J. Affect. Disord. 2020;275:48-57. https://doi.org/10.1016/j. jad.2020.06.022

24. Muller AE, Hafstad EM, Himmels JPW, Smedslund G, Flottorp S, Stensland S, et al. The mental health impact of the covid-19 pandemic on healthcare workers, and interventions to help them: A rapid systematic review, Psychiatry Research. 2020; 293: 113441, ISSN0165-1781. https:// doi.org/10.1016/j.psychres.2020.113441

25. Amatriain-Fernández S, Murillo-Rodríguez ES, Gronwald T, Machado S, Budde H. Benefits of physical activity and physical exercise in the time of pandemic. Psychological Trauma: Theory, Research, Practice, and Policy. 2020; 12:ed. S1. doi: 10.1037/tra0000643

26. Kang L, Li Y, Hu S, Chen M, Yang C, Yang BX, et al. The mental health of medical workers in Wuhan, China dealing with the 2019 novel coronavirus. Lancet Psychiatr. 2020;7(3):e14. doi: $10.1016 /$ S2215-0366(20)30047-X

27. Afonso P, Figueira ML. COVID-19 Pandemic: What are the Mental Health Risks? Revista Portuguesa de Psiquiatria e Saúde Mental. 2020;6(1):2-3. https://www.revistapsiquiatria.pt/index. php/sppsm/article/view/131

28. Roy A, Druker S, Hoge EA, Brewer JA. Physician Anxiety and Burnout: Symptom Correlates and a Prospective Pilot Study of App-Delivered Mindfulness Training JMIR Mhealth Uhealth. 2020;8(4):e15608. doi: 10.2196/15608 